\title{
EDGE DOMINATING GRAPH OF A GRAPH
}

\author{
B. BASAVANAGOUD ${ }^{1}$ AND SUNILKUMAR M. HOSAMANI ${ }^{2}$
}

\begin{abstract}
The edge dominating graph $E_{D}(G)$ of a graph $G=(V, E)$ is a graph with $V\left(E_{D}(G)\right)=$ $E(G) \cup S(G)$, where $S(G)$ is the set of all minimal edge dominating sets of $G$ with two vertices $u, v \in V\left(E_{D}(G)\right)$ adjacent if $u \in E$ and $v$ is a minimal edge dominating set of $G$ containing $u$. In this paper, we establish the bounds on order and size, diameter and vertex(edge) connectivity.
\end{abstract}

\section{Introduction}

All graphs considered here are finite, nontrivial, undirected, connected, without loops or multiple edges or isolated vertices. For undefined terms or notations in this paper, may be found in Harary [1].

Let $G=(V, E)$ be a graph. A set $S \subseteq E$ is an edge dominating set of $G$, if every edge in $E-S$ is adjacent to at least one edge in $S$. The edge domination number $\gamma^{\prime}(G)$ of $G$ is the minimum cardinality of an edge dominating set.

The minimal dominating graph of $G$ is an intersection graph on the minimal dominating sets of vertices of $G$. This concept was introduced by Kulli and Janakiram [4].

In [5], the concept of common minimal dominating graph of $G$ was defined as the graph having same vertex set as $G$ with two vertices adjacent if there is a minimal dominating set containing them. The concept of vertex minimal dominating graph $M_{V} D(G)$ of $G$ was introduced in [6], as the graph having $V\left(M_{V} D(G)\right)=V(G) \cup S(G)$, where $S(G)$ is the set of all minimal dominating sets of $G$ with two vertices $u, v$ adjacent if they are adjacent in $G$ or $v=D$ is a minimal dominating set containing $u$.

In this paper, we introduce the concept of edge dominating graph $E_{D}(G)$ of $G$ as the graph with $V\left(E_{D}(G)\right)=E(G) \cup S(G)$, where $S(G)$ is the set of all minimal edge dominating sets of $G$ with two vertices $u, v \in V\left(E_{D}(G)\right)$ adjacent, if $u \in E$ and $v=S$ is a minimal edge dominating set containing $u$.

In Figure 1, a graph $G$ and its edge dominating graph $E_{D}(G)$ are shown.

Corresponding author: B. Basavanagoud.

2010 Mathematics Subject Classification. 05C69.

Key words and phrases. Diameter, vertex connectivity, edge connectivity, domination number. 

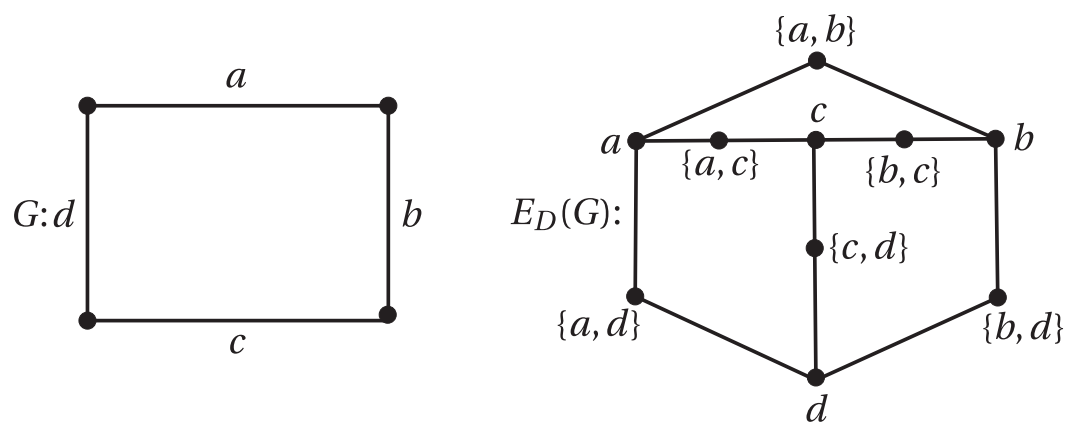

Figure 1:

\section{Preliminary results}

We need the following theorems for our further results.

Remark 1. For any graph $G, E_{D}(G)$ is bipartite.

Remark 2. $V\left(E_{D}(G)\right)=E \cup S$, where $S$ is the set of all minimal edge dominating sets of $G$. No two edges of $G$ and $S(G)$ are adjacent vertices in $E_{D}(G)$.

Remark 3. Let $G$ be a $(p, q)$-graph. Then $\operatorname{deg}_{E_{D}(G)}\left(e_{i}\right)=$ number of minimal edge dominating sets containing $e_{i}$, where $i=1,2, \ldots, q$.

Theorem A [3]. If G is a graph without isolated edges, then for every minimal edge dominating set $S, E-S$ is also an edge dominating set.

\section{Main results}

First, we obtain necessary and sufficient conditions on $G$ for which $E_{D}(G)$ is connected.

Theorem 3.1. For any graph $G$ with at least one edge, the edge dominating graph $E_{D}(G)$ of $G$ is connected if and only if $\Delta^{\prime}(G)<q-1$, where $\Delta^{\prime}(G)$ is the maximum edge degree of $G$.

Proof. Suppose there is no minimal edge dominating set containing both $x$ and $y$. Then there exists an edge $z \in E$ not adjacent to both $x$ and $y$. Let $S$ and $S^{\prime}$ be two maximal edge independent sets containing the edges $x z$ and $y z$ respectively. Since every maximal edge independent set is a minimal edge dominating set, therefore $x$ and $y$ are connected in $E_{D}(G)$ by a path $\left(x, S, z, S^{\prime}, y\right)$. Thus, $E_{D}(G)$ is connected. From this it follows that for any two edges $x, y \in E(G)$ either there exists a minimal edge dominating set containing $x$ and $y$ or there exist two nondisjoint minimal edge dominating sets $S_{1}$ and $S_{2}$ containing $x$ and $y$ respectively. This implies that in $E_{D}(G), x$ and $y$ are connected by a path of length at most four. 
Conversely, suppose $E_{D}(G)$ is connected. On the contrary $\Delta^{\prime}(G)=q-1$ and $x$ is an edge of degree $q-1$. Then $S=x$ is a minimum edge dominating set of $G$. Since every minimum edge dominating set is a minimal edge dominating set and further $G$ has at least two edges with $x$ adjacent to every other edge of $G, G$ has no isolated edges. Thus by Theorem A, $E-S$ contains a minimal edge dominating set $S^{\prime}$. Since $S \cap S^{\prime}=\phi$, in $E_{D}(G)$ there is no path joining $x$ to any edge of $E-S$. This implies that $E_{D}(G)$ is disconnected, a contradiction. Hence $\Delta^{\prime}(G)<q-1$.

In the next result, we obtain the bounds on the order of $E_{D}(G)$.

Theorem 3.2. For any graph $G$

$$
q+d^{\prime}(G) \leq p^{\prime} \leq \frac{q(q+1)}{2}
$$

where $d^{\prime}(G)$ is the edge domatic number of $G$ and $p^{\prime}$ denotes the number of vertices in $E_{D}(G)$. Further, the lower bound is attained if and only if $G=K_{1, p-1}$ and upper bound is attained if and only if $G$ is $q-2$ regular graph.

Proof. The lower bound follows from the fact that every graph has at least $d^{\prime}(G)$ number of minimal edge dominating sets of $G$ and the upper bound follows from the fact that every edge is in $(q-1)$ minimal edge dominating sets of $G$.

Suppose the lower bound is attained. Then every edge is in exactly one minimal edge dominating set of $G$ and hence, every minimal edge dominating set of $G$ is independent. This implies the necessity.

Suppose the upper bound is attained. Then each edge is in exactly $(q-1)$ minimal edge dominating sets and hence $G$ is $(q-2)$ regular. This implies the sufficiency.

In the following result, we obtain the bounds on the size of $E_{D}(G)$.

Theorem 3.3. For any graph $G$

$$
q \leq q^{\prime} \leq q(q-1)
$$

where $q^{\prime}$ is the number of edges in $E_{D}(G)$. Further, the lower bound is attained if and only if $G=K_{1, p-1}$ and the upper bound is attained if and only if $G$ is $a(q-2)$ regular graph.

Proof. Proof follows from Theorem 3.2.

Theorem 3.4. For any graph $G$

$$
\alpha_{0}\left(E_{D}(G)\right)=\min \{|E|,|S|\},
$$

where $S$ is the set of all minimal edge dominating sets of $G$. 
Proof. Let $V\left(E_{D}(G)=E \cup S\right.$. By Remark 2, $E$ and $S$ are independent sets and every element of $E$ is adjacent with some elements of $S$. Also we observe that $|E|<|S|$, then elements of $E$ covers all the edges of $E_{D}(G)$. Otherwise, elements of $S$ covers all edges of $E_{D}(G)$. Hence $\alpha_{0}\left(E_{D}(G)\right)=\min \{|E|,|S|\}$.

Theorem 3.5. For any graph $G$

$$
\beta_{0}\left(E_{D}(G)\right)=\max \{|E|,|S|\},
$$

where $S$ is the set of all minimal edge dominating sets of $G$.

Proof. For any graph $G$, clearly $E$ and $S$ are independent sets of $E_{D}(G)$. If $|E|>|S|$, then $\beta_{0}\left(E_{D}(G)\right)=|V|$. Otherwise $\beta_{0}\left(E_{D}(G)\right)=|S|$.

$$
\beta_{0}\left(E_{D}(G)\right)=\max \{|E|,|S|\} .
$$

Theorem 3.6. For any graph $G$ with $\Delta^{\prime}(G)<q-1$.

$$
\operatorname{diam}\left(E_{D}(G)\right) \leq 5
$$

where diam $(G)$ is the diameter of $G$.

Proof. By Theorem 3.1, $E_{D}(G)$ is connected. Let $u, v \in V\left(E_{D}(G)\right)$. We consider the following cases:

Case 1. Suppose $u, v \in E(G)$, then by Theorem 3.1, $d(u, v)_{E_{D}(G)} \leq 4$, where $d(u, v)_{E_{D}(G)}$ is the distance between $u$ and $v$ in $E_{D}(G)$.

Case 2. Suppose $u \in E(G)$ and $v \in S(G)$. Then $v=S$ is a minimal edge dominating set of $G$. If $u \in S$, then $u$ and $v$ are adjacent in $E_{D}(G)$. Otherwise there exist a vertex $w \in S$. This implies

$$
d(u, v)_{E_{D}(G)} \leq d(u, w)_{E_{D}(G)}+d(w, S)_{E_{D}(G)} \leq 4+1=5
$$

Case 3. Suppose $u, v \in S(G)$. Then $u=S_{1}$ and $u=S_{2}$ are two minimal edge dominating sets of $G$. If $S_{1}$ and $S_{2}$ are nonadjacent, then $d(u, v)_{E_{D}(G)}=d\left(S_{1}, S_{2}\right)_{E_{D}(G)}=2$. Otherwise there exist a minimal edge dominating set $S_{3}$ containing the vertices of $S_{1}$ and $S_{2}$. Thus

$$
d(u, v)_{E_{D}(G)}=d\left(S_{1}, S_{2}\right)_{E_{D}(G)} \leq d\left(S_{1}, S_{3}\right)_{E_{D}(G)}+d\left(S_{3}, S_{2}\right)_{E_{D}(G)} \leq 2+2=4 .
$$

Thus from all the above cases we get $\operatorname{diam}\left(E_{D}(G) \leq 5\right.$.

Theorem 3.7. For any graph $G$

$$
\gamma\left(\overline{E_{D}(G)}\right)=2 .
$$


Proof. Let $x \in E$ and $S$ be a minimal edge dominating set containing $x$. Then $x$ and $S$ together form a minimal edge dominating set of $\overline{E_{D}(G)}$. Since $\Delta\left(\overline{E_{D}(G)}\right) \leq p-2$, the result holds.

Theorem 3.8. For any graph $G, E_{D}(G)$ is bicolorable.

Proof. For any graph $G, E_{D}(G)$ is bipartite by Remark 1. Since chromatic number of any bipartite graph is two, therefore $E_{D}(G)$ is bicolorable.

Theorem 3.9. For any graph $G$

$$
\kappa\left(E_{D}(G)\right)=\min \left\{\min _{1 \leq i \leq q}\left(\operatorname{deg}\left(e_{i}\right)\right), \min _{1 \leq j \leq n}\left|S_{j}\right|\right\},
$$

where $S_{j}^{\prime}$ are the minimal edge dominating sets of $G$.

Proof. Let $G$ be a $(p, q)$ graph. Clearly edge set and minimal edge dominating sets of $G$ are independent. We consider the following cases:

Case 1. Let $x \in e_{i}$ for some $i$, having minimum edge degree among all $e_{i}^{\prime} s$ in $E_{D}(G)$. If the degree of $x$ is less than any other edge in $E_{D}(G)$, then by deleting those edges of $E_{D}(G)$ which are adjacent with $x$, results in a disconnected graph.

Case 2. Let $y \in S_{j}$ for some $j$ having minimum degree among all vertices of $S_{j}^{\prime} s$. If degree of $y$ is less than any other vertex in $E_{D}(G)$. Then by deleting those vertices which are adjacent with $y$, results in a disconnected graph. Thus $\kappa\left(E_{D}(G)\right)=\min \left\{\min _{1 \leq i \leq q}\left(\operatorname{deg}\left(e_{i}\right)\right), \min _{1 \leq j \leq n}\left|S_{j}\right|\right\}$.

Theorem 3.10. For any graph $G$

$$
\lambda\left(E_{D}(G)\right)=\min \left\{\min _{1 \leq i \leq q}\left(\operatorname{deg}\left(e_{i}\right)\right), \min _{1 \leq j \leq n}\left|S_{j}\right|\right\}
$$

where $S_{j}^{\prime} s$ are the minimal edge dominating sets of $G$.

Proof. Follows from the same lines in the proof Theorem 3.9.

\section{Acknowledgement}

${ }^{1}$ No.F.510/1/DRS/2010(SAP-I) dated: August 2010.

2 This research was supported by the University Grants Commission, New Delhi,India-No.F.43/2006(BSR)/7-101/2007(BSR) dated: September 2009. 


\section{References}

[1] F. Harary, Graph Theory, Addison-Weseley, Reading Mass, 1969.

[2] T.W.Haynes, S.T.Hedetniemi and P. J. Slater, Fundamentals of Domination in Graphs, Marcel Dekker, Inc, New York, 1998.

[3] S. R. Jayaram, Line domination in graphs, Graphs Combin., 3(1987), 357-363.

[4] V. R. Kulli and B. Janakiram, The minimal dominating graph, Graph Theory Notes of New York, New York, 28(1995), 12-15.

[5] V. R. Kulli and B. Janakiram, The common minimal dominating graph, Indian J. Pure. Appl. Math., 27(1996), 193-196.

[6] V. R. Kulli, B. Janakiram and K. M. Niranjan, The vertex minimal dominating graph, Acta Ciencia Indica, 28(2002), 435-440.

[7] V. R. Kulli, B. Janakiram and K. M. Niranjan, The dominating graph, Graph Theory Notes of New York, 46(2004), 5-8.

Department of Mathematics, Karnatak University, Dharwad-580 003, Karnataka, India.

E-mail: b.basavanagoud@gmail.com

E-mail: bgouder1@yahoo.co.in 